ИЗВЕСТИЯ АКАДЕМИИ НАУК ЭСТОНСКОП ССР. ФИЗИКА * МАТЕМАТИКА

\title{
ОДНА ОЦЕНКА ТОЧНОСТИ ПРИБЛИЖЕНИЯ ИНТЕГРАЛЬНЫХ ЭКСПОНЕНТ КВАДРАТУРНЫМИ ФОРМУЛАМИ
}

\author{
(Представил Г. Кузмин)
}

\section{Введение}

При приближенном решении уравнения переноса часто требуется аппроксимировать интегральные экспоненты $E_{p}, p \geqslant 1$, квадратурными формулами (КФ) [ $\left.{ }^{1}\right]$, и быстрота сходимости метода зависит от того насколько хорошо мы можем это делать.

В данной работе для широкого класса КФ выводится левосторонняя эценка приближения $E_{p}$. Эта оценка связывает степень аппроксимации $E_{p}$ с первым узлом КФ и позволяет доказать неулучшаемость оценок скорости сходимости некоторых методов решения уравнения переноса (в частности, метода дискретных ординат $\left.\left[{ }^{2}\right]\right)$.

Рассмотрим последовательность КФ, аппроксимирующих интеграл

$$
\begin{gathered}
\int_{0}^{1} f(\mu) \mathrm{d} \mu \approx \sum_{j=1}^{n} \alpha_{j} f\left(\mu_{j}\right), \\
\alpha_{j} \geqslant 0, j=1,2, \ldots, n ; 0<\mu_{1}<\mu_{2}<\ldots<\mu_{n} \leqslant 1, n=1,2, \ldots .
\end{gathered}
$$

План работы следующий. В первом разделе выводится асимптотическое равенство для соотношения $\sum_{j=1}^{n} \alpha_{j} / \mu_{j}^{k}, k=1,2, \ldots$, при помощи которого во втором разделе доказывается, что интегральную экспоненту $E_{p}$ при помощи КФ (1), удовлетворяющей некоторым естественным требованиям, нельзя приблизить лучше, чем $\mu_{1}^{p-1}$, где $\mu_{1}-$ первый узел КФ. Третий раздел работы посвящен обсуждению этих требований.

Обозначим через $c, c_{k}, c_{k, l}$ положительные постоянные, которые могут принимать различные значения в различных соотношениях.

\section{1. Веса и узлы КФ}

Предположим, что КФ (1) удовлетворяет для любого $n$ следующим требованиям
A) $\mu_{l} \leqslant \sum_{j=1}^{l} \alpha_{j} \leqslant \mu_{l+1}, \quad l=1,2, \ldots, n, \mu_{n+1}=1$,
B) $\mu_{l+1} \leqslant c \mu_{l}, \quad l=1,2, \ldots, n$,
C) $c_{1}<\sum_{j=1}^{n} \alpha_{j}\left(\frac{1}{\mu_{j}}-\frac{1}{\mu_{j+1}}\right)<c_{2}$ 
Обозначим $J=\left[1 / \mu_{1}\right], J^{+}=J+1, J^{-}=J-1$ и введем целіочисленные множества

$$
\begin{gathered}
N_{i}^{+}=\left\{k>0: \mu_{k+1} \leqslant \frac{1}{\imath}\right\} ; \quad N_{i}=\left\{k>0: \mu_{k} \leqslant \frac{1}{\imath}\right\} \\
N_{i}^{-}=\left\{k>0: \mu_{k-1} \leqslant \frac{1}{\imath}\right\}
\end{gathered}
$$

для $i=1,2, \ldots, J^{+}$, положив $\mu_{0}=0$. Очевидно, что $\quad N_{J^{+}}^{+}=N_{J^{+}}=\varnothing$, а $N_{J^{+}}=\{1\}$. Пуусть далее

$$
I_{i}^{+}=\sum_{j \in N_{i}^{+}} \alpha_{j} ; \quad I_{i}=\sum_{j \in N_{i}} \alpha_{j} ; \quad I_{i}^{-}=\sum_{j \in N_{i}^{-}} \alpha_{j} .
$$

Нетрудно видеть, что $0 \leqslant I_{i}^{+} \leqslant I_{i} \leqslant I_{i}^{-}, i=1, \ldots, J^{+}$и

$$
\begin{gathered}
I_{1}^{+}=I_{1}=I_{1}^{-}=\sum_{j=1}^{n} \alpha_{j}, \\
I_{J^{+}}^{+}=I_{J^{+}}=0, \quad I_{J^{+}}^{-}=\alpha_{1} .
\end{gathered}
$$

Из условий А) и В) следуют неравенства

$$
\begin{aligned}
& I_{i}^{+} \leqslant \mu_{l+1} \leqslant \frac{1}{i}, \quad l \in N_{i}^{+}, \quad l+1 \notin N_{i}^{+}, \\
& I_{i} \geqslant \mu_{l}>\frac{1}{i}, \quad l \in N_{i}^{-}, \quad l+1 \notin N_{i}^{-}, \\
& I_{i} \geqslant \mu_{l} \geqslant c \mu_{l+1}>\frac{c}{i}, \quad l \in N_{i}, l+1 \notin N_{i}, \\
& I_{i} \leqslant \mu_{l+1} \leqslant c \mu_{l} \leqslant \frac{c}{i}, \quad l \in N_{i}, \quad l+1 \notin N_{i} .
\end{aligned}
$$
ства

Рассмотрим теперь как связаны $I_{i}{ }^{+}, I_{i}$ и $I_{i}{ }^{-}$. Справедливы равен-

$$
I_{i}^{+}=I_{i}-\alpha_{l(i)}
$$

где

$$
\alpha_{l(i)}= \begin{cases}\alpha_{l}, & l \in N_{i}, l \notin N_{i}^{+}, \\ 0, & N_{i}=N_{i}^{+},\end{cases}
$$

и

$$
I_{i}^{-}=I_{i}+\bar{\alpha}_{l(i)}
$$

где

$$
\bar{\alpha}_{l(i)}= \begin{cases}\alpha_{l}, & l \in N_{i}^{-}, l \notin N_{i}, \\ 0, & N_{i}=N_{i}^{-} .\end{cases}
$$

Таким образом, $\alpha_{l(i)}$ - это вес КФ, соответствующий узлу $\mu_{l(i)}$ такому, что $\mu_{l(i)} \leqslant \frac{1}{i}$, но $\mu_{l(i)+1}>\frac{1}{i}$ и соответственно $\bar{\alpha}_{l(i)}$ такой вес, что $\mu_{l(i)-1} \leqslant \frac{1}{i}, \mu_{l(i)}>\frac{1}{i}$. Заметим, что 


$$
\sum_{i=1}^{j} \alpha_{l(i)}=\sum_{j=1}^{n} m_{j} \alpha_{j}
$$

где

$$
m_{j}=\left\{k^{\prime}-k \geqslant 0: \frac{1}{k+1}<\mu_{j+1} \leqslant \frac{1}{k}, \frac{1}{k^{\prime}+1}<\mu_{j} \leqslant \frac{1}{k^{\prime}}\right\},
$$

г. е. $m_{j}$ равно числу промежутков $\left(\frac{1}{k}, \frac{1}{k+1}\right), \quad$ на которое $j$-й узел КФ опережает $j+1$-й узел. Аналогично

$$
\sum_{i=1}^{J} \bar{\alpha}_{l(i)}=\sum_{j=1}^{n} \bar{m}_{j} \alpha_{j}
$$

где $\bar{m}_{j}=m_{j-1}, j=2, \ldots, n, \bar{m}_{1}=0$. Отсюда

$$
\sum_{j=1}^{n} m_{j}=J^{-}
$$

a

$$
\sum_{j=1}^{n} \bar{m}_{j}=J--m_{n}
$$

Из неравенств

$$
\frac{1}{k+1}<\mu_{j+1} \leqslant \frac{1}{k}, \quad \frac{1}{k^{\prime}+1}<\mu_{j} \leqslant \frac{1}{k^{\prime}}
$$

следует, что

$$
\frac{1}{\mu_{j}}-\frac{1}{\mu_{j+1}}-1 \leqslant k^{\prime}-k \leqslant \frac{1}{\mu_{j}}-\frac{1}{\mu_{j+1}}+1 \text {, }
$$

откуда

$$
\begin{aligned}
\sum_{j=1}^{n} \alpha_{j}\left(\frac{1}{\mu_{j}}-\frac{1}{\mu_{j+1}}\right)-\sum_{i=1}^{n} \alpha_{j} & \leqslant \sum_{i=1}^{J} \alpha_{l(i)}=\sum_{j=1}^{n} m_{j} \alpha_{j} \leqslant \\
& \leqslant \sum_{j=1}^{n} \alpha_{j}\left(\frac{1}{\mu_{j}}-\frac{1}{\mu_{j+1}}\right)+\sum_{j=1}^{n} \alpha_{j} .
\end{aligned}
$$

Теперь, учитывая условия А) и С), заключаем, что существуют такие постоянные $c_{1}$ и $c_{2}$, что справедливо неравенство

$$
c_{1}<\sum_{i=1}^{J} \alpha_{l(i)}<c_{2}
$$

Аналогичное неравенство имеет место и для суммы $\vec{\alpha}_{l(i)}$.

Теперь сформулируем и докажем основной результат этого раздела. Л е м а. Пусть КФ удовлетворяет условиям А)-С). Тогда справедливо равенство

$$
\sum_{j=1}^{n} \frac{\alpha_{j}}{\mu_{j}^{k}}=\left\{\begin{array}{l}
c_{n}^{1}+\left|\ln \mu_{1}\right|, \quad k=1, \\
c_{n}^{k} \mu_{1}^{1-k}+O\left(\mu_{1}^{2-k}\right), \quad k>1,
\end{array}\right.
$$

причем $c_{n}^{k} \rightarrow c_{0}^{k}, n \rightarrow \infty, k=1,2, \ldots$.

Доказ а тельство. Рассмотрим отдельно случаи $k=1$ и $k>1$. При $k=1$ можно выписать следующую цепочку (мы используем технику, развитую в $\left.\left[{ }^{3}\right]\right)$ 


$$
\begin{aligned}
\sum_{j=1}^{n} \frac{\alpha_{j}}{\mu_{j}} & =\sum_{i=1}^{J} \sum_{j \in N_{i} \backslash N_{l+1}} \alpha_{j} / \mu_{j} \leqslant \sum_{i=1}^{J}(i+1) \sum_{j \in N_{i} \backslash N_{t+1}} \alpha_{j}= \\
& =\sum_{i=1}^{J}(i+1)\left(I_{i}-I_{i+1}\right)=\sum_{i=1}^{J}(i+1) I_{i}-\sum_{i=1}^{J}(i+1) I_{i+1}= \\
& =\sum_{i=1}^{J}(i+1) I_{i}-\sum_{i=2}^{J^{+}} i I_{i}=2 I_{1}-J+I_{J}+\sum_{i=2}^{J} I_{i}^{(2),(3)}= \\
& =\sum_{j=1}^{n} \alpha_{j}+\sum_{i=1}^{J} I_{i}{ }^{A), 8)} \leqslant 1+\sum_{i=1}^{J} I_{i}+\sum_{i=1}^{J} \alpha_{l(i)} \stackrel{(4),(10)}{\leqslant} \\
& \leqslant 1+c+\sum_{i=1}^{J} \frac{1}{i}=c_{J}^{1}+\ln J,
\end{aligned}
$$

где $c_{J}^{1} \rightarrow c_{0}^{1}, n \rightarrow \infty$. (Здесь и далее над знаками равенств и неравенств стоят номера используемых условий и соотношений).

Покажем обратное неравенство. Имеем

$$
\begin{aligned}
\sum_{j=1}^{n} \alpha_{j} / \mu_{j} & =\sum_{i=1}^{J} \sum_{j \in N_{i} \backslash N^{N_{t+1}}} \alpha_{j} / \mu_{j} \geqslant \sum_{i=1}^{J} i \sum_{j \in N_{i} \bigwedge^{N_{t+1}}} \alpha_{j}= \\
& =\sum_{i=1}^{J} i\left(I_{i}-I_{i+1}\right)=\sum_{i=1}^{J} i I_{i}-\sum_{i=1}^{J} i I_{i+1}= \\
& =\sum_{i=1}^{J} i I_{i}-\sum_{i=1}^{J^{+}}(i-1) I_{i}=-J I_{J}+\sum_{i=1}^{J} I_{i}^{(3),(9)}= \\
& =\sum_{i=1}^{J} I_{i}-\sum_{i=1}^{J} \bar{\alpha}_{l(i)}{ }^{(5),(10)} \sum_{i=1}^{J} 1 / i-c=\bar{c}_{J}^{1}+\ln J
\end{aligned}
$$

где $\bar{c}_{J}^{1} \rightarrow \bar{c}_{0}^{1}, n \rightarrow \infty$. Таким образом, при $k=1$ лемма доказана.

Пусть теперь $k>1$. Тогда учитывая неравенства

$$
\begin{aligned}
& (i+1)^{k}-i^{k} \leqslant k(i+1)^{k-1}, \\
& i^{k}-(i-1)^{k} \geqslant k(i-1)^{k-1},
\end{aligned}
$$

получим

$$
\begin{aligned}
\sum_{j=1}^{n} \alpha_{j} / \mu_{j}^{k} & =\sum_{i=1}^{J} \sum_{j \in N_{i} \backslash N_{i+1}} \alpha_{j} / \mu_{j}^{k} \geqslant \sum_{i=1}^{J} i^{k}\left(I_{i}-I_{i+1}\right)= \\
& =\sum_{i=1}^{J} i^{k} I_{i}-\sum_{i=1}^{J^{+}}(i-1) I_{i}= \\
& =\sum_{i=1}^{J}\left[i^{k}-(i-1)^{k}\right] I_{i}+(J+-1)^{k} I_{J^{+}}{ }^{(3),(13)} \\
& \geqslant k \sum_{i=1}^{J}(i-1)^{k-1} I_{i}>^{(6)} k c \sum_{i=1}^{J} \frac{(i-1)^{k-1}}{i} \geqslant \\
& \geqslant c_{n}^{k} J^{k-1}+O\left(J^{k-2}\right) .
\end{aligned}
$$

Обратное неравенство показывается аналогично, применяя вместо (6) a (13) соответственно неравенства (7) и (12).

Принимая во внимание, что $J=\left[1 / \mu_{1}\right]$, приходим к равенствам (11). Лемма доказана. 


\section{2. Аппроксимация интегральных экспонент при помощи КФ}

Будем приближать интегральные экспоненты $E_{p}$, определяемые по формуле $\left[{ }^{1}\right]$

$E_{p}(\tau)=\int_{1}^{\infty} \frac{\exp (-v \tau)}{v^{p}} \mathrm{~d} v=\int_{0}^{1} \frac{\exp (-\tau / \mu)}{\mu^{2-p}} \mathrm{~d} \mu, \quad p=2,3, \ldots, \tau \geqslant 0$,

последовательностью КФ (1), удовлетворяющих требованиям А)-C). Положим дополнительно, что КФ точны для многочленов степени $p-2$, г. е. имеет место условие

D)

$$
\sum_{j=1}^{n} \alpha_{j} \mu_{j}^{k}=\frac{1}{k+1}, \quad k=0,1, \ldots, p-2 .
$$

Найдем асимптотическое разложение $E_{p}$. Применяя несколько раз равенство [ $\left.{ }^{4}\right]$

$$
E_{p}^{\prime}(\tau)=-E_{p-1}(\tau), \quad p=2,3, \ldots,
$$

получим цепочку равенств

$$
\begin{aligned}
& E_{p}(\tau)=E_{p}(0)-\int_{0}^{\tau} E_{p-1}\left(\tau^{\prime}\right) \mathrm{d} \tau^{\prime}=E_{p}(0)-\tau E_{p-1}(0)+ \\
& +\int_{0}^{\tau} \int_{0}^{\tau^{\prime}} E_{p-2}\left(\tau^{\prime \prime}\right) \mathrm{d} \tau^{\prime \prime} \mathrm{d} \tau^{\prime}=\ldots= \\
& =E_{p}(0)-\tau E_{p-1}(0)+\ldots+(-1)^{p-1} \frac{\tau^{p-3^{*}}}{(p-3) !} E_{3}(0)+ \\
& +(-1)^{p} \underbrace{\int_{0}^{\tau} \ldots \int_{0}^{\tau}}_{p-2} E_{2}(\tau) \underbrace{\mathrm{d} \tau \ldots \mathrm{d} \tau}_{p-2} .
\end{aligned}
$$

Далее, используя известное разложение $\left[{ }^{5}\right]$

$E_{1}(\tau)=\int_{0}^{1} \frac{\exp (-\tau / \mu)}{\mu} \mathrm{d} \mu=-\ln \tau-\gamma+\tau-\frac{\tau^{2}}{2 \cdot 2 !}+\frac{\tau^{3}}{3 \cdot 3 !}-\ldots \quad(\tau>0)$, где $\gamma$ - постоянная Эйлера, получаем для малых :

$$
E_{2}(\tau)=E_{2}(0)+\int_{0}^{\tau} E_{1}^{\prime}\left(\tau^{\prime}\right) \mathrm{d} \tau^{\prime}=1+\tau(\ln \tau-1+\gamma)+O\left(\tau^{2}\right)
$$

Подставляя значение $E_{2}$ в (15), приходим к равенству

$$
\begin{aligned}
& E_{p}(\tau)=\sum_{i=1}^{p-2}(-1)^{i-1} \frac{\tau^{i-1}}{(p-i)(i-1) !}+(-1)^{p} \frac{\tau^{p-2}}{(p-2) !}+
\end{aligned}
$$

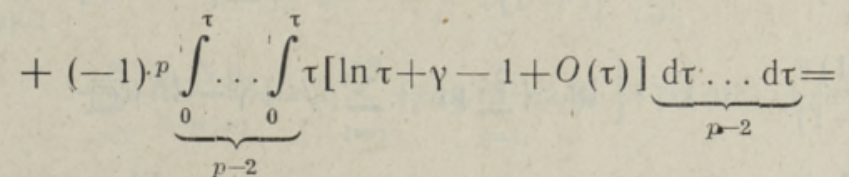

$$
\begin{aligned}
& =\sum_{i=1}^{p-1} \frac{(-1)^{i-1} \tau^{i-1}}{(p-i)(i-1) !}+\frac{(-1)^{p}}{(p-1) !} \tau^{p-1}\left[\ln \tau-\sum_{i=1}^{p-1} 1 / i+\gamma\right]+O\left(\tau^{p}\right) .
\end{aligned}
$$


О人бозначим

$$
E_{p}^{n}(\tau)=\sum_{j=1}^{n} \alpha_{j} \mu_{j}^{p-2} \exp \left(-\tau / \mu_{j}\right), \quad p=2,3, \ldots ; \tau \geqslant 0 .
$$

Раскладывая экспоненту в ряд, получим

$$
\begin{aligned}
E_{p}^{n}(\tau) & =\sum_{j=1}^{n} \alpha_{j} \mu_{j}^{p-2}\left(1-\frac{\tau}{\mu_{j}}+\ldots(-1)^{p} \frac{\tau^{p-2}}{(p-2) ! \mu_{j}^{p-2}}\right)+ \\
& +\sum_{j=1}^{n} \alpha_{j} \mu_{j}^{p-2} \cdot \frac{(-1)^{p-1}}{(p-1) !} \tau^{p-1}\left(\frac{1}{\mu_{j}^{p-1}}-\frac{\tau}{p \mu_{j}^{p}}+\frac{\tau^{2}}{p(p+1) \mu_{j}^{p+1}}-\ldots\right)= \\
& =\sum_{i=1}^{p-1} \frac{(-1)^{i}}{(i-1) !} \tau^{i-1} A_{n}^{i}-\frac{(-1)^{p}}{(p-1) !} \tau^{p-1} A_{n}^{p}(\tau),
\end{aligned}
$$

где

$$
A_{n}^{i}=\sum_{j=1}^{n} \alpha_{j} \mu_{j}^{p-i-1}, \quad i=1,2, \ldots, p-1,
$$

$A_{n}^{p}(\tau)=\sum_{j=1}^{n} \frac{\alpha_{j}}{\mu_{j}}-\frac{\tau}{p}\left\{\sum_{j=1}^{n} \frac{\alpha_{j}}{\mu_{j}^{2}}-\frac{\tau}{p+1} \sum_{j=1}^{n} \frac{\alpha_{j}}{\mu_{j}^{3}}+\frac{\tau^{2}}{(p+1)(p+2)} \sum_{j=1}^{n} \frac{\alpha_{j}}{\mu_{j}^{4}}-\ldots\right\}$.

В последнем равенстве обозначим $s=\tau / \mu_{1}$. Тогда, учитывая результат леммы, имеем

$$
\begin{aligned}
A_{n}^{p}(\tau) & =c_{n}^{1}+\left|\ln \mu_{1}\right|-\frac{s}{p}\left\{c_{n}^{2}+O(1)-\frac{s}{p+1}\left[c_{n}^{3}+O(1)\right]+\right. \\
& \left.+\frac{s^{2}}{(p+1)(p+2)}\left[c_{n}^{4}+O(1)\right]-\ldots\right\}= \\
& =-\ln \mu_{1}+\left\{c_{n}^{1}-\frac{c_{n}^{2}+O(1)}{p} s+\frac{c_{n}^{3}+O(1)}{p(p+1)} s^{2}-\right. \\
& \left.-\frac{c_{n}^{4}+O(1)}{p(p+1)(p+2)} s^{3}+\ldots\right\}=-\ln \mu_{1}+d_{n}^{p}(s)
\end{aligned}
$$

причем

$d_{n}^{p}(s) \rightarrow d_{0}^{p}(s) \equiv c_{0}^{1}-\frac{c_{0}^{2}+O(1)}{p} s+\frac{c_{0}^{3}+O(1)}{p(p+1)} s^{2}-\frac{c_{0}^{4}+O(1)}{p(p+1)(p+2)} s^{3}+\ldots$ при $n \rightarrow \infty$.

Сопоставим теперь асимптотические разложения $E_{p}$ и $E_{p}{ }^{n}$, определенные в (16) и (18) соответственно. Имеем

$$
\begin{aligned}
& E_{p}\left(s \mu_{1}\right)-E_{p}^{n}\left(s \mu_{1}\right)=\sum_{i=1}^{p-1} \frac{(-1)^{i-1}}{(i-1) !}\left(s \mu_{1}\right)^{i-1}\left[\frac{1}{p-i}-A_{n}^{i}\right]+ \\
& +\frac{(-1)^{p}}{(p-1) !}\left(s \mu_{1}\right)^{p-1}\left[\ln s+\ln \mu_{1}-\sum_{i=1}^{p-1} 1 / i+\gamma-\ln \mu_{1}+\right. \\
& \left.\quad d_{n}^{p}(s)\right]+O\left[\left(s \mu_{1}\right)^{p}\right] .
\end{aligned}
$$

Принимая во внимание условие D), получим, что $A_{n}{ }^{i}=1 /(p-i)$ и 


$$
\bar{E}_{p}\left(s \mu_{1}\right)-E_{p}^{n}\left(s \mu_{1}\right)=\frac{(-1)^{p}}{(p-1) !}\left(s \mu_{1}\right)^{p-1}\left[\ln s+\bar{d}_{n}^{p}(s)\right]+O\left[\left(s \mu_{1}\right)^{p}\right],
$$

где

$$
\bar{d}_{n}^{p}(s)=d_{n}^{p}(s)+\gamma-\sum_{i=1}^{p-1} 1 / i \rightarrow d_{0}^{p}(s)+\gamma-\sum_{i=1}^{p-1} 1 / i, \quad n \rightarrow \infty .
$$

Теперь зафиксируем $s \in(0,1]$ так, чтобы $\ln s+\bar{d}_{n}^{p}(s) \neq 0$. На возможность этого указывает тот факт, что функция $\ln s+\bar{d}_{0}^{p}(s)$ суть аналитическая неравная тождественно нулю. Значит существует $a_{p}=$ $=$ const $>0$ такая, что

$$
\left|E_{p}\left(s \mu_{1}\right)-E_{p}^{n}\left(s \mu_{1}\right)\right| \geqslant a_{p} \mu_{1}^{p-1}, \quad n \geqslant n_{0} .
$$

Итак, справедлива

Т е о р ем а. Пусть КФ удовлетворяет условиям А)-D). Тогда справедлива оценка

$$
\max _{0 \leqslant \tau \leqslant \infty}\left|E_{p}(\tau)-E_{p}^{n}(\tau)\right| \geqslant a_{p} \mu_{1}^{p-1}, \quad n \geqslant n_{0}, \quad a_{p}=\text { const }>0,
$$

əде $\mu_{1}$ - первый узел КФ, а функции $E_{p}$ и $E_{p}{ }^{n}$ определяются соответственно равенствами (14) $и$ (17).

3 а м е ч н и е. Проводя аналогичным образом аппроксимацию функции $\tau^{l} E_{p}(\tau), l \geqslant 0$, можно показать, что имеет место оценка

$$
\max _{\tau \geqslant 0} \mid \tau^{l}\left(E_{p}(\tau)-E_{p}^{n}(\tau) \mid \geqslant a_{p}^{l} \mu_{1}^{p+l-1}, \quad n \geqslant n_{0}^{\prime}, \quad l \geqslant 0, \quad a_{p}^{l}=\text { const }>0 .\right.
$$

\section{3. Обсуждение КФ}

В этом разделе на примере некоторых КФ рассмотрим выпслнение условий А) -D).

Прежде, чем проверить выполнение условий В) и С), отметим, что эти условия не связаны между собой, а именно:

У т в ер ждение 1. Существуют КФ, удовлетворяющие А) $и$ В), но неудовлетворяющие С).

У тв ер ждени е 2. Существуют КФ, удовлетворяющие А) $и$ С), но неудовлетворяющие В).

В качестве доказательства приведем примеры таких формул. КФ, узлы и веса которой есть

$$
\mu_{j}=a^{j-1} \mu_{1}, \quad \alpha_{j}=a^{j-1 / 2} \mu_{1}, \quad j=1,2, \ldots, n, \quad a \geqslant 2,
$$

удовлетворяет А) и В). Действительно,

$$
\mu_{l}=a^{l-1} \mu_{1} \leqslant \sum_{j=1}^{l} \alpha_{j}=\mu_{1} \frac{\sqrt{a}}{a-1}\left(a^{l}-1\right) \leqslant a^{l} \mu_{1}=\mu_{l+1}
$$

и

Однако

$$
\mu_{l+1} \leqslant a_{\mu l}, \quad l=1,2, \ldots, n .
$$

$$
\sum_{j=1}^{n} \frac{\alpha_{j} \Delta \mu_{j}}{\mu_{j} \mu_{j+1}}=(1-1 / a) \sum_{j=1}^{n} \alpha_{j} / \mu_{j}=\sqrt{a}(1-1 / a) n \rightarrow \infty
$$

при $n \rightarrow \infty$. (Здесь и далее $\left.\Delta \mu_{j}=\mu_{j+1}-\mu_{j}, j=1, \ldots, n\right)$.

С другой стороны, КФ

$$
\mu_{1}=1 / n^{2}, \quad \alpha_{1}=1,5 / n^{2}, \quad \mu_{j}=(j-1) / n, \quad \alpha_{j}=1 / n, \quad j=2,3, \ldots, n
$$


не удовлетворяет условию В), ибо $\mu_{2} / \mu_{1}=n$. Но

$$
\mu_{l}=\frac{l-1}{n}<\sum_{j=1}^{l} \alpha_{j}=\frac{1,5}{n^{2}}+\frac{l-1}{n}<\frac{l}{n}=\mu_{l+1}
$$

H

$$
\sum_{j=1}^{n} \frac{\alpha_{j} \Delta \mu_{i}}{\mu_{j} \mu_{j+1}}=1,5(1-1 / n)+\sum_{j=2}^{n} \frac{1}{j(j-1)}<c .
$$

Утверждения 1 и 2 доказаны.

1) КФ средних прямоугольников:

$$
\alpha_{j}=1 / n, \quad \mu_{j}=(j-1 / 2) / n, \quad j=1,2, \ldots, n .
$$

Выполнение А) и В) очевидно. Далее из равенства

$$
\sum_{j=1}^{n} \alpha_{j}\left(\mu_{j}^{-1}-\mu_{j+1}^{-1}\right)=\sum_{j=1}^{n} \frac{1}{(j-1 / 2)(j+1 / 2)}
$$

следует С). Условие D) выполнимо для $p \leqslant 3$.

2) КФ Гаусса, отображенные на $[0,1]$. Выполнение А) немедленно следует из теоремы Чебышева-Маркова-Стильтьеза [ $\left.{ }^{6}\right]$. Условие В) проверено в $\left[^{3}\right]$, а D) имеет место при $p \leqslant 2 n+1$. Для проверки С) заметим, что веса КФ Г аусса можно представить в виде

$$
\alpha_{j}=(\pi / 2 n) \sin \theta_{j}+O\left(1 / n^{2}\right), \quad j=1,2, \ldots, n,
$$

где $\left[{ }^{5}\right]$

$$
\theta_{j}=\frac{j-1 / 4}{n+1 / 2} \pi+\left(8 n^{2}\right)^{-1} \operatorname{ctg} \frac{j-1 / 4}{n+1 / 2} \pi+O\left(n^{-3}\right) .
$$

Представление (19) можно получить, применяя к выражению для весов КФ Гаусса асимптотическую формулу Дарбу для производных от многочленов Лежандра $\left[{ }^{6}\right]$. Теперь нетрудно доказать С). Из (19)-(20) следует, что

$$
\alpha_{j} \Delta \mu_{j} \leqslant \frac{c}{n^{2}} \sqrt{\mu_{j}}\left[\sqrt{\mu_{j}}+1 / n\right], \quad j=1,2, \ldots, n
$$

и отсюда

$$
\sum_{j=1}^{n} \alpha_{j}\left(\mu_{j}^{-1}-\mu_{j+1}^{-1}\right) \leqslant(c / n) \sum_{j=1}^{n} 1 / \mu_{j} \leqslant c_{1} .
$$

Обратное неравенство получается без труда.

3) КФ Кленшоу-Куртиса, отображенные на $[0,1]$. Известно, [ $\left.{ }^{7}\right]$, что узлы и веса этих КФ имеют вид

$$
\begin{gathered}
\mu_{j}=\sin ^{2}(\pi j / 2 n), \quad j=1,2, \ldots, n, \quad n-\text { четное. } \\
\alpha_{j}=(2 / n) \sum_{i=0}^{n / 2}\left(1-4 i^{2}\right)^{-1} \cos (2 \pi i j / n), \quad j=1,2, \ldots, n-1, \\
\alpha_{n}=0,5 /\left(n^{2}-1\right) .
\end{gathered}
$$

(Здесь $\Sigma^{\prime \prime}$ означает, что первый и последний члеі взяты с коэффициенroм 0,5$)$.

В $\left[{ }^{8}\right]$ доказано, что

$$
\alpha_{j}=(\pi / 2 n) \sin (\pi j / n)+O\left(n^{-4} \sin ^{-2}(\pi j / n)\right), \quad j=1,2, \ldots, n-1,
$$

откуда следует выполнение А) и С). Выполнение В) очевидно, а D) 
справедливо для $p \leqslant n+2$, что следует из построения КФ. КленшоуКуртиса.

4) Составная КФ. Разделим отрезок $[0,1]$ на $m+1$ отрезков точками

$$
0<a_{0}<a_{1}<\ldots<a_{m+1}=1 .
$$

Применяя на каждом отрезке $\left[a_{j}, a_{j+1}\right], j=0,1, \ldots, m$ КФ с узлами $\xi_{i} \in(0,1]$ и весами $\omega_{i}>0, i=1,2, \ldots, n$, получим узлы $\mu_{k}$ и веса $\alpha_{k}, k=1,2, \ldots,(m+1) n$ новой $\mathrm{K} \Phi$, а именно $\left[{ }^{3}\right]$ :

$$
\left\{\begin{array}{l}
\mu_{j n+i}=a_{j}+\xi_{i} \Delta a_{j}, \quad \Delta a_{j}=a_{j+1}-a_{j}, \\
\alpha_{j n+i}=\omega_{i} \Delta a_{j}, \quad j=0,1, \ldots, m, \quad i=1,2, \ldots, n .
\end{array}\right.
$$

Предположив, что $\xi_{i}, \omega_{i}, i=1,2, \ldots, n$ удовлетворяют требованиям A) - D), можно показать, что и КФ с узлами и весами (21) удовлетворяет этим условиям.

\section{ЛИТЕРА Т У А}

1. Кейз К., Цвайфель П. Линейная теория переноса. М., «Мир», 1972.

2. Вайникко Г. М., Мариак А. Л. Изв. ВУЗов. Математика, № 11, 11-22 (1978).

3. Pitkäranta, J., Scott, L. R. SIAM J. Numer. Anal., 20, 922 (1983).

4. Смелов В. В. Лекции по теории переноса нейтронов. М., Атомиздат, 1978.

5. Абрамовіч М. Н., Стиган Н. Справочник по специальным функциям с формуламн, графиками и математическими таблицами. М., «Наука», 1979.

6. Сеге $Г$. Ортогональные многочлены. М., Физматгиз, 1962,

7. Imhof, I. P. Numer. Math, № 5, 138-141 (1963).

8. Мариак А. Л. Изв. АН ЭССР. Физ. Матем., 35, № 3, 338-340 (1986).

\section{Институт астрофизики и физики атмосферы Академии наук Эстонской СССР}

Поступила в редакцию 10/XI 1985

Переработанный вариант 21/II 1986

\section{A. MARSAK}

\section{UKS INTEGRAALSETE EKSPONENTIDE KVADRATUURVALEMITEGA LÄHENDAMISE VEAHINNANG}

Käesolevas töös on tuletatud vasakpoolne integraalsete eksponentide kvadratuurvalemitega lähendamise veahinnang. Töestatakse, et integraalset eksponenti $E_{p}$ ei saa lähendada ühtlases meetrikas paremini kui const $\mu_{1}{ }^{p-1}$, kus $\mu_{1}$ on esimene sōlm kvadratuurvalemis, mis rahuldab tingimusi - A) - D). On kontrollitud nende tingimuste täidetust mõningate (tavaliselt kasutatavate) kvadratuurvalemite jaoks.

\section{A. MARSHAK}

\section{AN ESTIMATE OF THE APPROXIMATION $\Lambda$ CCURACY OF THE INTEGRAL EXPONENTS BY QUADRATURE RULES}

In the present paper the left-hand estimate of the approximation accuracy of the integral exponents $E_{p}, p>1$ by quadrature rules is deduced for a wide'class of quadrature rules. This estimate connects the accuracy of the approximation of $E_{p}$ with the first point of quadrature rule and permits to prove the nonimprovement of the estimates of the convergence rate of some methods for solving the transport equation (for example, the discrete ordinates method).

The plan of this paper is as follows: in section 1 the asymptotic equality for $\stackrel{n}{\Sigma} \alpha_{j} / \mu_{j}{ }^{h}, k=1,2, \ldots ; n=1,2, \ldots$ is derived. Here $\alpha_{j}=\alpha_{j}(n) \geqslant 0, \mu_{j}=\mu_{j}^{(n)} \in[0,1], j=$ $=1, \ldots, n$ are quadrature weights and points. In section 2 it is proved that one cannot approximate the integral exponents $E_{p}, p>1$ by the quadrature rule better than $\mu_{1}{ }^{p-1}$, where $\mu_{1}$ is the first point of quadrature rule that satisfies some of natural conditions, These conditions are discussed in the third section. 